Meeting report

\title{
Second International Symposium on the Molecular Biology of Breast Cancer, March 12-16, 2000, Lillehammer, Norway Chris Jones and Giske Ursin*
}

\author{
University College London, London, UK and *USC/Norris Comprehensive Cancer Center, Los \\ Angeles, California, USA
}

Received: 24 March 2000

Accepted: 27 March 2000

Published: 13 April 2000 (c) Current Science Ltd

5-10\% of all early onset (age below 40 years) breast cancer patients have mutations in BRCA1 or BRCA2. Weber presented data demonstrating how breast cancer penetrance in BRCA1 mutation carriers varies with the population studied - from $87 \%$ by age 85 years in high-risk families to $50 \%$ by age 69 years or even lower in population-based samples.

Both Weber and Bruce Ponder (Cambridge University, Cambridge, UK) emphasized that penetrance may vary with the site of the mutation, and that families with mutations in the middle region of BRCA2 may have at least reduced breast cancer penetrance, if not higher ovarian cancer incidence. Ponder reminded us that knowledge of the mutation status of the family may also be of significance for male family members. The risk of prostate and colon cancer are threefold and fourfold elevated in BRCA1 mutation carriers, whereas the risk of prostate and pancreas cancer is elevated threefold in BRCA2 mutation carriers. The data presented here provide a strong rationale for expanding $B R C A 1$ and BRCA2 testing at institutions where the counselling expertise is available. A number of prevention trials are in progress, which hopefully will soon yield useful information to individuals with identified mutations. Mike Stratton (Institute of Cancer Research, Surrey, UK) described the pathology of inherited breast tumours. He presented data suggesting that, although BRCA1/BRCA2 tumours cannot be diagnosed microscopically, they tend to be of higher grade than sporadic tumours. There was also a slight increase in medullary tumours in $B R C A 1$ mutation carriers. Immunohistochemically, BRCA1 (but not BRCA2) tumours are less likely to be oestrogen receptor (ER)-, progesterone receptor- and p53-positive than are sporadic tumours. Both $B R C A 1$ and BRCA2 tumours have a lower degree of $\mathrm{c}^{-}$ erbB2-positive tumours, whereas cyclin $D$ is more likely to be upregulated in $B R C A 1$ than in $B R C A 2$ tumours. 
Åke Borg (Lund University, Lund, Sweden) further described data from comparative genomic hybridization (CGH) studies suggesting that there are distinct differences between BRCA1, BRCA2 and other familial cancers. Preliminary results from cDNA microarray analyses suggest that $B R C A 1$ and $B R C A 2$ have distinct gene expression profiles, which include genes that are involved in cell cycle control. Both Borg and Stratton suggested that there may be additional undiscovered major breast cancer susceptibility genes. There are a number of non$B R C A 1 / B R C A 2$ familial cases of ductal cancers with lower grade of mitosis that are ER-positive and progesterone-receptor-positive, as well as cases of invasive lobular cancer, and $\mathrm{CGH}$ analyses suggest that these tumours have distinct patterns of chromosomal alterations.

Manfred Schwab (German Cancer Center, Heidelberg, Germany) ended the session with intriguing late-breaking news suggesting that there may be a BRCA2-modifying gene or a fragile site on 9p23-24. Data from three unlinked families with male breast cancer suggest that this is a region of frequent aberrations.

\section{Hormonal influence on breast cancer: what is new?}

The role of coactivators in the action of oestrogen was summarized by Myles Brown (Dana Farber Institute, Boston, MA, USA). He reminded the participants that the effect of endocrine therapy is limited and specific responses may be determined by the ER coregulators that are expressed. Important coactivators include p300/ CREB binding protein, and the steroid receptor coactivator-1 family. Results presented further suggest that growth factors can help modulate oestrogen activity through activation of mitogen-activated protein kinase. AlB1, a member of the steroid receptor coactivator- 1 family that is amplified in ER-positive breast cancers, can be activated by mitogen-activated protein kinase, which further causes the activation of p300 and histone acetyltransferase activity.

Kathryn Horwitz (University of Colorado, Denver, CO, USA) gave an excellent talk on the role of progesterone. Recent evidence suggests that postmenopausal hormone replacement therapy with oestrogen and a synthetic version of progesterone increases the risk of breast cancer. The rate and dose of progesterone administration is important, and determines whether there will be growth stimulation or inhibition. Continuous progesterone treatment appears to inhibit growth. Data was also presented on the two progesterone receptors $A$ and $B$ in different types of tissue. Progesterone receptors $A$ and $B$ are coexpressed, but the ratio varies from tissue to tissue. In humans there is an excess of type $B$ receptors, and in human breast cancer there is a large variation in the ratio of type A to type $B$ receptors. This could influence the responses of the tumour to progesterone, because a number of genes appear to be differ- entially regulated by type $A$ and type $B$ receptors. Oestrogen upregulates both type $A$ and type $B$ receptors. Horwitz suggested that these different types of receptors may provide the basis for selective progesterone receptor modulators, new progesterone ligands that target only one progesterone receptor or one tissue. The distribution of type $A$ versus that of type $B$ receptors may also be important as a prognostic marker in breast cancer.

Suzanne Fuqua (Baylor College, Houston, TX, USA) described expression array experiments using RNA from tamoxifen-sensitive and tamoxifen-resistant MCF-7 tumours isolated from athymic mouse xenograft models. The data suggest specific tamoxifen-resistant RNA profiles, with an endothelial receptor-related protein overexpressed in these tumours. These intriguing results are in accord with what is known about the role of angiogenesis/intratumoural microvessel density in breast cancer prognosis. Jan Åke Gustafsson (Karolinska Institute, Huddinge, Sweden) gave an inspiring talk about the 'Yin-Yang' effects of ER $\alpha$ and ER $\beta$. In rodent uterus $17 \beta$-oestradiol downregulates ER $\beta$ and upregulates ER $\alpha$. In mammary gland of rodents, ER $\alpha$ is high before puberty, low in pregnancy, high in lactation and low after lactation. ER $\beta$ on the other hand is present at all times. ER $\beta$ seems to quench the activity of ER $\alpha$ in both rodent mammary and uterine tissue.

Mitchell Dowsett (Royal Marsden Hospital, London, UK) ended the session with an interesting talk on the necessity of intermediate markers of endocrine responsiveness. Clinical trials usually measure response in terms of complete or partial response, stable disease and progressive disease. Dowsett suggested that we should be satisfied with stable disease, which translates to no tumour growth and no change in cell proliferation.

\section{Growth factor receptors}

The type I receptor tyrosine kinases and their ligands are expressed at high levels in breast tumours, and are starting to provide novel targets for therapy. José Baselga (Hospital General Universitari Vall d'Hebron, Barcelona, Spain) focused on anticancer strategies aimed at the epidermal growth factor (EGF) receptor (ErbB1, HER1). Anti-EGF receptor monoclonal antibody 225 directed against the extracellular domain of EGF receptor has been found to enhance greatly the effects of chemotherapeutic agents that are active against breast cancer, such as taxol and doxorubicin, in vitro. Phase I clinical trials on a human:murine chimeric antibody (C225) have demonstrated its efficacy in patients with head and neck cancer, especially in combination with cisplatin. Another target for therapy is the tyrosine kinase activity of EGF receptor, and a new family of potent tyrosine kinase inhibitors have shown antitumour activity against breast cancer cell lines. Data from phase I clinical trials of ZD1839 were presented for the first time, ahead of a full presentation at the Ameri- 
can Society of Clinical Oncology meeting in May. In that trial, at least a partial response was seen in up to $20 \%$ of patients with multiple tumour types, and in vivo inhibition of receptor function was demonstrated, but skin and gastrointestinal toxicity was observed in a proportion of cases.

The Herceptin story (Genentech Inc, San Francisco, USA), from the production of the humanized antibody through to the early phase I clinical trials in 1990-1991, and up to future trials that are currently seeking approval, was well covered by Dennis Slamon (University of California, Los Angeles, CA, USA) and Charles Vogel (University of Miami School of Medicine, Miami, FL, USA). Of particular interest were the controversial studies, due to start this year, on the use of Herceptin in the adjuvant setting. From the floor, Per Eystein Lønning (Haukeland Hospital, Bergen, Norway) questioned the direction of such trials when the majority of even the strongest ErbB2 overexpressors failed to respond to Herceptin alone or in combination with traditional chemotherapeutic agents, and wondered where the translational research to explain this was going to come from.

An interesting talk to close the session came from Klaus Pantel (University Hospital Eppendorf, Hamburg, Germany), who presented a strong association of micrometastatic breast tumour cells in the bone marrow with ErbB2 positivity, opening the possibility of a more effective immunotherapeutic target than overt metastases.

\section{The role of TP53 and ATM in breast cancer}

The ATM/TP53 pathways are fundamental in the cell's response to DNA damage, and dysregulation of these pathways play a crucial part in the development of human cancer. New insights into the mechanisms of these pathways are opening up exciting possibilities for selective nongenotoxic cancer chemotherapeutic strategies.

Tak Mak (Ontario Cancer Institute, Toronto, Ontario, Canada) focused on the recently identified checkpoint kinase (chk)2, which directly phosphorylates p53 in response to $\gamma$-radiation-induced DNA damage. Chk2-/embryonic stem cells failed to maintain $\gamma$-irradiation-induced arrest in the $\mathrm{G}_{2}$ phase of the cell cycle, and $\mathrm{Chk}^{-/-}$thymocytes were resistant to DNA damage-induced apoptosis.

David Lane (University of Dundee, Dundee, UK) concentrated on the p53 modulator mdm2, and the potential for anti-mdm2 monoclonal antibodies or antisense oligonucleotides as nongenotoxic chemotherapeutic agents. He also described the upstream inhibitor of $\mathrm{mdm} 2$, Arf, which is a potent activator of the $p 53$ response.

Curtis Harris (National Cancer Institute, Bethesda, MA, USA) provided evidence for the contribution of two DNA helicases, XPB and XPD, which are associated with xeroderma pigmentosum, toward p53-mediated apoptosis.
Data implicating other DNA helicases associated with Werner syndrome and Bloom syndrome in p53-mediated apoptosis were also presented.

Other talks in this session included that by Scott Lowe (Cold Spring Harbor Laboratory, New York, NY, USA), who described p53 action in apoptosis and senescence, and suggested that selection against apoptosis during tumour development can give rise to drug-resistant tumours.

Richard Gatti (University of California, Los Angeles, CA, USA) and Janet Hall (International Agency for Research on Cancer, Lyon, France) discussed the increased cancer susceptibility and radiation sensitivity of ataxia telangiectasia gene (ATM) heterozygotes, and argued for two populations of ATM heterozygotes with different phenotypes.

\section{Cell cycle and apoptosis}

Although much time was devoted to the ATM/TP53 pathways, and their associations with cell cycle control and apoptosis, these two topics were confined to single presentations in their own right.

Jiri Bartek (Danish Cancer Society, Copenhagen, Denmark) examined the restriction point switch in late $G_{1}$ phase of the cell cycle, and addressed the question regarding whether the RB-E2F pathway was the only physiological way to commit mammalian cells to DNA replication. Evidence was presented for a parallel myc-controlled pathway, which is independent of and co-operates with the RB pathway.

The role of apoptosis in breast cancer was presented by Charles Streuli (University of Manchester, Manchester, UK), who highlighted cell adhesion as a dominant signal for the survival of epithelial cells in the breast. The removal of adhesion signals from cells in culture leads to apoptosis, and the basement membrane was shown to play a critical role in cell survival via the integrin class of receptors and focal adhesion kinases, which signal to the $\mathrm{Bcl}-2$ family of apoptosis-regulating proteins. An important point was raised in the discussion about the breast epithelial cells commonly used in culture without attachment to an extracellular matrix; it was pointed out that the majority of commercially available 'normal mammary epithelial cells' are not derived from the luminal epithelium, but are in fact myoepithelial cells.

\section{Angiogenesis and telomerase activity}

Microvessel density as an independent prognostic factor in breast cancer has highlighted the importance of angiogenesis in the development of tumours. Adrian Harris (Imperial Cancer Research Fund, Oxford, UK) examined the role of hypoxia in switching on angiogenesis by the activation of vascular endothelial growth factor. Two hypoxia inducible transcription factors (hif1 and hif2) have been identified as mediating two different hypoxia-regu- 
lated pathways. A novel target for gene therapy has been identified downstream of hif - the carbonic anhydrase $\mathrm{CA} 9$ - which appears to regulate tumour $\mathrm{pH}$ and may be associated with drug resistance.

The telomerase story was eloquently covered by Jerry Shay (University of Texas, Dallas, TX, USA). The emergence of telomerase inhibitors as potential breast cancer chemotherapeutic agents has been strengthened by results showing the induction of apoptosis in immortalized human breast epithelial cells by antisense therapies. In the clinical setting, it is suggested that telomerase inhibitors may be most beneficially used after surgery in combination with chemotherapy/radiotherapy, and/or synergistically with other compounds such as antiangiogenic factors.

\section{How to implant our knowledge of the molecular biology of breast cancer into the clinic?}

Jean-Marc Nabholtz (University of Alberta, Edmonton, Canada) opened the session with a historic overview of chemotherapy in breast cancer, and further outlined some of the current issues with newer combined regimens.

Jan GM Klijn (Dr Daniel den Hoed Cancer Clinic, Rotterdam, The Netherlands) described factors that predict response to endocrine therapy. High ER and progresterone receptor levels are associated with greater response to tamoxifen, whereas high p53 expression is associated with poorer response to tamoxifen. HER2/neu is a weak prognostic factor in breast cancer, but a strong predictor for lack of response to endocrine therapy. Klijn presented data showing that the lowest response to tamoxifen is found in the group with p53 mutations and lowest ER levels. He also described a breast cancer activity resistance locus (BCAR-1) on 16q; tumours with low level of BCAR-1 have higher response rate with tamoxifen.

Per Eystein Lønning (Haukeland University Hospital, Bergen, Norway) ended the session with an inspiring talk on whether we are using the right approaches in clinical trials. He suggested that we ought to limit the testing of new cocktails, and instead use the biology of the tumour to guide us to the proper therapy. Furthermore, he underlined the advantages of using sequential rather than combined therapy; this should facilitate the use of predictive factors and reduce toxicity.

This session clearly outlined how basic science can be integrated into the clinic, and perhaps also, as the last speaker mentioned, what the clinic can add to basic science.

\section{New technologies in defining the tumour genotype}

The closing session of the meeting concentrated on some of the new molecular techniques that look set to greatly facilitate investigations into breast cancer biology.
The development of tissue microarrays to enable highthroughput molecular profiling was presented by Olli Kallioniemi (National Institutes of Health, Bethesda, MA, USA). The production of a 'tissue chip' containing 500-1000 core biopsies of individual tumours allows the examination of DNA, RNA and protein targets in a large number of tissues in single experiments. The issue of whether small foci of breast tumours truly reflect the whole specimen, given breast tumour heterogeneity, was raised, but evidence was presented that suggest that perhaps this heterogeneity may not be as important as was thought.

Joe Gray (University of California, San Francisco, CA, USA) detailed molecular cytogenetic approaches to analyze genome evolution in human breast cancers. CGH investigates the population average of tumour cells, and evidence was presented to indicate that the 'average' tumour genotype arises early and changes slowly during cancer progression. The analysis of individual cells carried out using fluorescent in situ hybridization paradoxically suggest a drastic cell-cell variability in genome content, leading to the interpretation that the production of a cell with an advantageous genome change may be an extremely rare event in breast tumourigenesis.

Finally David Botstein (Stanford University, Stanford, California, USA) discussed genome-wide investigations using cDNA microarrays. By utilizing clustering analysis of expressed genes from a series of breast cancers, distinct subtypes of tumours could be identified with important implications for prognosis and treatment. Differences in gene expression from the nonepithelial cells in the tumours could also be analyzed using this approach. Furthermore, investigations of breast cancer cell lines reiterated the point that these cultures may be derived either from the luminal or myoepithelial cells, with very different patterns of gene expression. There can be little doubt that array technology is going to open up a vast wealth of data in the coming years, and the challenge for cancer biologists is going to be in the analysis and interpretation of this data. George Klein (Karolinska Institute, Stockholm, Sweden) summed this up by stating that we 'must learn to love complexity'.

\section{Conclusion}

This was an excellent conference, bringing together an impressive array of internationally distinguished speakers to provide a thorough state-of-the-art review of the basic molecular science of breast cancer and its applications in the clinic. The number of speakers who presented new and unpublished data was a testament to the regard held for the organizers and quality of the meeting in general. It is to be hoped that the goal of promoting collaborations will also bear fruit. 
Authors' affiliations: Chris Jones (Breast Molecular Pathology Group, Department of Histopathology, Royal Free and University College Medical School, University College London, London, UK) and Giske Ursin (Assistant Professor, Department of Preventive Medicine, USC/Norris Comprehensive Cancer Center, Los Angeles, California, USA)

Correspondence: Dr Chris Jones, Breast Molecular Pathology Group, Department of Histopathology, Royal Free and University College Medical School, University College London, Rockefeller Building, University Street, London WC1E 6JJ, UK. Tel: +44 (0)20 7679 6014; fax: +44 (0)20 7387 3674; e-mail: cjones@ucl.ac.uk 